ARTICLE

\title{
Multiple recent horizontal transfers of a large genomic region in cheese making fungi
}

Kevin Cheeseman ${ }^{1,2,3, \star}$, Jeanne Ropars $4,5,6, \star$, Pierre Renault ${ }^{1,3}$, Joëlle Dupont ${ }^{4}$, Jérôme Gouzy $7,8,9$, Antoine Branca ${ }^{5,6}$, Anne-Laure Abraham ${ }^{1,3}$, Maurizio Ceppi ${ }^{2}$, Emmanuel Conseiller², Robert Debuchy ${ }^{10,11}$, Fabienne Malagnac ${ }^{10,12}$, Anne Goarin ${ }^{10}$, Philippe Silar ${ }^{10,12}$, Sandrine Lacoste ${ }^{4}$, Erika Sallet $7,8,9$, Aaron Bensimon², Tatiana Giraud ${ }^{5,6} \&$ Yves Brygoo ${ }^{13}$

While the extent and impact of horizontal transfers in prokaryotes are widely acknowledged, their importance to the eukaryotic kingdom is unclear and thought by many to be anecdotal. Here we report multiple recent transfers of a huge genomic island between Penicillium spp. found in the food environment. Sequencing of the two leading filamentous fungi used in cheese making, $P$. roqueforti and $P$. camemberti, and comparison with the penicillin producer $P$. rubens reveals a $575 \mathrm{~kb}$ long genomic island in $P$. roqueforti-called Wallaby-present as identical fragments at non-homologous loci in P. camemberti and P. rubens. Wallaby is detected in Penicillium collections exclusively in strains from food environments. Wallaby encompasses about 250 predicted genes, some of which are probably involved in competition with microorganisms. The occurrence of multiple recent eukaryotic transfers in the food environment provides strong evidence for the importance of this understudied and probably underestimated phenomenon in eukaryotes.

\footnotetext{
${ }^{1}$ INRA, UMR1319 Micalis, F-78352 Jouy-en-Josas, France. ${ }^{2}$ Genomic Vision, 80-84 rue des Meuniers, 92220 Bagneux, France. ${ }^{3}$ AgroParisTech, UMR Micalis, F-78352 Jouy-en-Josas, France. ${ }^{4}$ Origine, Structure, Evolution de la Biodiversité, UMR 7205 CNRS-MNHN, Muséum National d'Histoire Naturelle, CP39, 57 rue Cuvier, 75231 Paris Cedex 05, France. ${ }^{5}$ Univ Paris-Sud, Ecologie, Systématique et Evolution, UMR8079, 91405 Orsay, France. ${ }^{6}$ CNRS, Ecologie, Systématique et Evolution, UMR8079, 91405 Orsay, France. ${ }^{7}$ LIMP Toulouse, INRA/CNRS, INRA, 24 Chemin de Borde Rouge-Auzeville, CS 52627,31326 Castanet-Tolosan Cedex, France. ${ }^{8}$ INRA, Laboratoire des Interactions Plantes-Microorganismes (LIPM), UMR441, F-31326 Castanet-Tolosan, France. ${ }^{9}$ CNRS, Laboratoire des Interactions Plantes-Microorganismes (LIPM), UMR2594, F-31326 Castanet-Tolosan, France. ${ }^{10}$ Univ Paris-Sud, Institut de Génétique et Microbiologie, UMR8621, 91405 Orsay, France. ${ }^{11}$ CNRS, Institut de Génétique et Microbiologie UMR8621, 91405 Orsay, France. ${ }^{12}$ Univ Paris Diderot, Sorbonne Paris Cité, Institut des Energies de Demain (IED), 75205 Paris, France. ${ }^{13} 13$ ruelle d'Aigrefoin 78470 St Rémy-lès-Chevreuse. * These authors contributed equally to the work. Correspondence and requests for materials should be addressed to T.G. (email: Tatiana.Giraud@u-psud.fr).
} 
$\mathrm{H}$ umans have created many novel, nutrient-rich and homogeneous environments exerting strong selection pressures and inducing the rapid adaptation of microorganisms, such as fungal pathogens of crops, food spoilers and domesticated fungi used for fermentation in beverage or food (for example, Saccharomyces for bread, beer and wine, Aspergillus for traditional fermented Asian foods, such as sake, soy sauce and miso, and Penicillium for cheese and cured or fermented meat). These rapid adaptations in fungi provide excellent models for studying general processes of eukaryotic genome evolution, including the functional and ecological impact of horizontal gene transfer ${ }^{1}$ and changes in metabolism ${ }^{2}$. Prokaryote-to-prokaryote transfers have been recognized as common and their associated impact important enough to raise questions about the possibility of reconstructing prokaryotic history through a tree of life or to change practices relating to antibiotic use in medicine. By contrast, horizontal transfers in eukaryotic species are still perceived by many to be isolated, sporadic events with a limited impact ${ }^{3-5}$. However, over the last 20 years or so, a number of cases of gene transfer in eukaryotes have been described. These documented cases include transfers of genetic material of prokaryotic origin into a eukaryote host ${ }^{6}$ and transfers in man-made environments, for example, between yeasts used for wine fermentation ${ }^{1}$ or pathogenic fungi on crops ${ }^{6-9}$. Both the sizes and number of genes involved vary widely. Transfers of a single gene, a complete metabolic pathway ${ }^{10}$, whole chromosomes ${ }^{11}$ or even cases of the integration of almost complete genomes from bacterial endosymbionts into their eukaryotic hosts $^{12}$ have been described. Notable impacts of recently described horizontal transfers include key roles in land colonization by plants $^{13}$, pigment production in spider mites through the acquisition of fungal carotenoid biosynthesis genes ${ }^{14}$ and the emergence of plant diseases through transfers in fungi ${ }^{15}$. Despite these examples, the lack of specific evolutionary trends in reported cases of lateral gene transfer in eukaryotes has led to the view of ancient, sporadic and isolated events with relatively little global impact on eukaryotic kingdoms, rather than a more frequently and widely occurring phenomenon. The frequency and importance of eukaryote-to-eukaryote gene transfer may, however, be underestimated.

Penicillium species are ubiquitous filamentous ascomycetes important to the biotechnology, biomedical and food industries. They commonly occur as food spoilage agents and opportunistic pathogens and are widely used as versatile cell factories. $P$. camemberti and $P$. roqueforti are used as starter cultures for cheeses. P. camemberti, used for the maturation of soft cheeses, such as Camembert, is the result of many selection programs aiming to improve the texture and colour of the conidia or physiological characteristics. $P$. camemberti has never been isolated from substrates other than dairy products. $P$. roquefort $i$ is widespread in food and also occurs in silage and natural environments. It is used as a starter culture in the production of most blue-veined cheeses (including Roquefort, Gorgonzola, Stilton and Danish Blue) and its abilities to tolerate cold temperatures, low oxygen concentrations, alkaline and weak acid preservatives, make it a common spoilage agent in refrigerated stored foods, meat products, rye bread and silage ${ }^{16,17}$. To our knowledge, despite the importance of the filamentous fungi used in cheese making, no genome sequence has yet been published for any of these species. The availability of these first two genome sequences will therefore provide a useful resource for improving our knowledge of edible cheese moulds and for comparative genomics.

Here we sequence and assemble the genomes of $P$. roquefort $i$ and $P$. camemberti and compare them with two other available Penicillium genomes, those of the penicillin producer and food spoilage agent $P$. rubens, previously known as $P$. chrysogenum ${ }^{18}$, and of the Citrus pathogen $P$. digitatum ${ }^{19}$. We report a case of multiple, present-day horizontal transfers of a very large (over $500 \mathrm{~kb}$ ) genomic island between several cheese fungi. This genomic island harbours about 250 genes, some of which are probably involved in competition with other microorganisms. Beyond the potential conceptual and applied implications of recurrent horizontal transfers occurring in food, this finding indicates that horizontal gene transfer (HGT) may be more widespread and important than previously thought in eukaryotes.

\section{Results}

Detection of a horizontally transferred genomic island. The global characteristics and comparisons of the genomes of $P$. camemberti, $P$. roquefort $i, P$. rubens and $P$. digitatum are given in Table 1. The genomes have similar sizes and number of genes, with the exception of $P$. digitatum, which has fewer genes than the other three genomes. This smaller number of genes is thought to be the result of a streamlining process affecting the genome of $P$. digitatum due to its specialized plant pathogenic lifestyle ${ }^{19}$. Assembly quality, as shown by the N50 metric and the number of scaffolds, is high for $P$. roqueforti and $P$. rubens. The initial in silico assembly for $P$. roquefort $i$ has been experimentally validated and further improved (see below). The genome assemblies for $P$. camemberti and $P$. digitatum appear more fragmented.

Surprisingly, several scaffolds from $P$. roquefort $i$, $P$. camembert $i$ and $P$. rubens had stretches of more than $5 \mathrm{~kb}$ displaying $100 \%$ identity in common (Supplementary Fig. S1), whereas mean pairwise identity was, otherwise, only $85-90 \%$ between genomes. $P$. digitatum completely lacked these regions that were identical in the other Penicillium species. We investigated the nature of these shared sequences, by locating these regions accurately in the genome of $P$. roqueforti by improving assembly quality. The availability of a high-quality genome sequence in this clade will also improve the resolving power of comparative genome analysis in subsequent studies. For this purpose, we used a combination of polymerase chain reaction (PCR) and molecular combing, a powerful fluorescent in situ hybridization-based technique for the direct visualization of single-DNA molecules ${ }^{20,21}$. Molecular combing resulted in the successful mapping of scaffolds, including some separated by more than a $100 \mathrm{~kb}$, onto single-DNA molecules, thus constituting a new means of improving or experimentally validating de novo genome assemblies (Supplementary Fig. S2). It yielded an assembly in which $92 \%$ of the $P$. roquefort $i$ genome was clustered into six super scaffolds of chromosomal sizes (Supplementary Fig. S3).

In the $P$. roqueforti FM164 strain, all the sequences found identical to both $P$. rubens and $P$. camemberti clustered together within a single $575 \mathrm{~kb}$ region accounting for $2 \%$ of the genome, which we called 'Wallaby' (Figs 1 and 2). This region lies within a $7.8 \mathrm{Mb}$ chromosome (Supplementary Fig. S3). The genomes of additional strains of $P$. roqueforti were examined by SOLiD resequencing. Three lacked the entire Wallaby region, whereas the fourth carried the very same Wallaby sequence as FM164. These sequences were aligned for precise mapping of the Wallaby insertion point (Fig. 3). Some of the regions flanking Wallaby could be characterized in $P$. rubens and $P$. camembert $i$ and were found to share $85-90 \%$ identity in the three species. However, these sequences were located on other scaffolds than those carrying the Wallaby fragments in the two later species (Fig. 2), indicating non-homologous locations of Wallaby between all three species. The possibility of misassembly yielding these different locations was excluded by the successful PCR amplification of fragments overlapping the edges of the identical sequences in the three genomes. Furthermore, rearrangement events appeared to have occurred after the transfers in all three species. 
Table 1 | Accession numbers and global characteristics of the Penicillium genomes compared in this study.

\begin{tabular}{|c|c|c|c|c|}
\hline Species & P. camemberti & P. roqueforti & P. rubens & P. digitatum \\
\hline Strain & FM 013 & FM164 & Wisconsin 54-1255 & PHI26 \\
\hline Substrate & Cheese environment & Cheese environment & Mouldy cantaloupe & Citrus contaminant \\
\hline Accession numbers EMBL & HG793134 to HG793313 & HG792015 to HG792062 & AM920416-AM920464 & JH993687-JH993786 \\
\hline Genome size $(\mathrm{Mb})$ & 34 & 28 & 32 & 26 \\
\hline Number of scaffolds & 181 & $\begin{array}{l}46 \text { (combing assembly) } \\
73 \text { (initial assembly) }\end{array}$ & 49 & 100 \\
\hline Minimum length (bp) & 2,008 & 2,101 & 1,032 & 964 \\
\hline GC content & 48.22 & 48.65 & 48.9 & 48.9 \\
\hline Number of protein-coding genes & 14,578 & 13,036 & 12,943 & 9,153 \\
\hline Mean gene length (bp) & $1,504.04$ & $1,812.71$ & 1,515 & 1,387 \\
\hline Per cent genes with introns & 77 & 81 & 83.5 & 67 \\
\hline Mean number of exons per gene & 2.96 & 3.09 & 3 & 2.66 \\
\hline
\end{tabular}

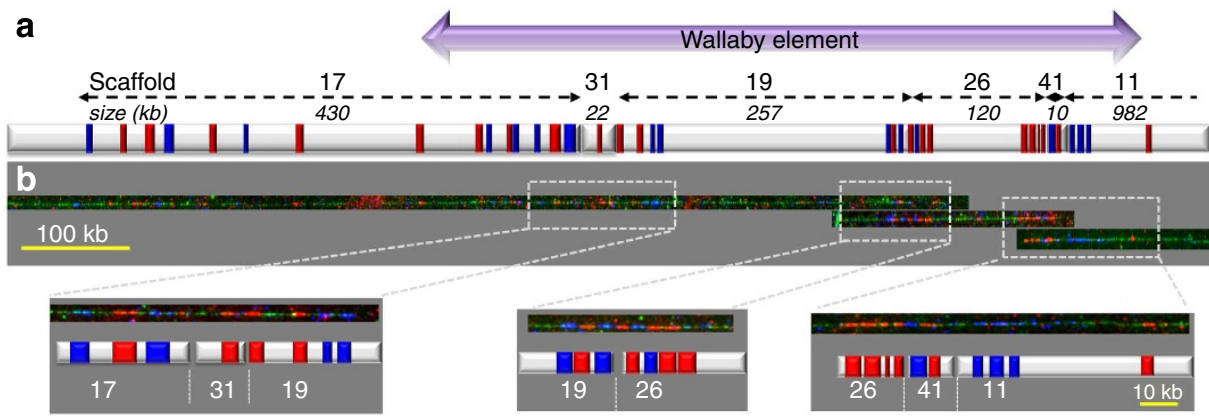

Figure 1 | Structural characterization of the Wallaby region in P. roqueforti FM164. (a) In silico genomic Morse code for physical mapping, by molecular combing, of the six scaffolds bearing the Wallaby element (purple arrow). The numbers at the top indicate the scaffold names, with sizes in kilobases (kb) indicated below. (b) Visualization of the scaffolds mapped to the Wallaby locus. Grey boxes show a higher magnification of scaffold junctions. Images are re-coloured based on fluorescence signal emission lengths. Green: YOYO-1 stained combed DNA fibre. Red: Probe detected with Alexa 594 conjugated antibodies. Blue: Probe detected using Alexa 394 and AMCA conjugated antibodies.

Indeed, the Wallaby sequences of $P$. rubens and $P$. camemberti lacked various fragments present in $P$. roqueforti, and both contained a $86-\mathrm{kb}$ fragment absent from $P$. roqueforti (Fig. 2).

Wallaby displayed little similarity to sequences from public databases, even those of the well-studied Aspergillus genus, the closest relative of Penicillium ${ }^{18}$. When Blast hits could be obtained, they matched fungal genomes (Supplementary Fig. S4), indicating a fungal origin for Wallaby. A tetranucleotide composition analysis of Wallaby and its comparison with several fungal genomes revealed a nucleotide composition of Wallaby that seemed to be different from the rest of the genomes of $P$. rubens, $P$. camemberti and $P$. roqueforti, but still closer from these genomes than any other (Table 2 and Supplementary Fig. S5). The nucleotide composition of Wallaby also differed from that of other available fungal genomes, but was nevertheless closest to Aspergillus and Penicillium strains, suggesting that the donor species lie within this clade (Table 2, Supplementary Fig. S5).

Overall, these results indicate that Wallaby has been recently and independently acquired, in at least some of these Penicillium species, via horizontal transfers. Other alternative explanations, such as introgression, can be excluded because of the non-homologous locations of the identical sequences in $P$. rubens and $P$. camemberti, precluding a parsimonious hybridization hypothesis. The perfect identity of the sequences also argues for very recent transfer events. No synonymous mutations were found in Wallaby, except for some repeat induced point (RIP) mutation footprints in $P$. roqueforti, indicating that the presence of Wallaby is not an ancestral character. Pairwise comparison of the genome-wide distribution of $100 \%$ identical sequences between $P$. roqueforti and the three other genomes revealed no long stretches of sequences identical in all three genomes other than those in Wallaby. The only variation within Wallaby thus corresponded to RIP mutation footprints in the $P$. roquefort $i$ Wallaby region (Fig. 2; Supplementary Fig. S6). In fungi, the RIP mechanism induces multiple C:G to T:A substitutions in repeated sequences during sex events ${ }^{22,23}$. As a consequence, sequence identity in Wallaby fragments with RIP footprints dropped to 90$97 \%$ between $P$. roqueforti and the other two Penicillium sequences, but this concerned exclusively RIP C:G to T:A substitutions. This is consistent with the occurrence of RIP after the transfer event in $P$. roqueforti.

The region flanking Wallaby in $P$. roqueforti may be a hotspot for DNA insertions, as other unrelated fragments appear to be inserted at this locus in other Penicillium genomes (Fig. 4). However, no footprints of duplication or transposable elements were found around the Wallaby insertion points for which flanking regions could be identified.

Wallaby transfer to several other species. The presence of Wallaby was further investigated in 441 strains by PCR 


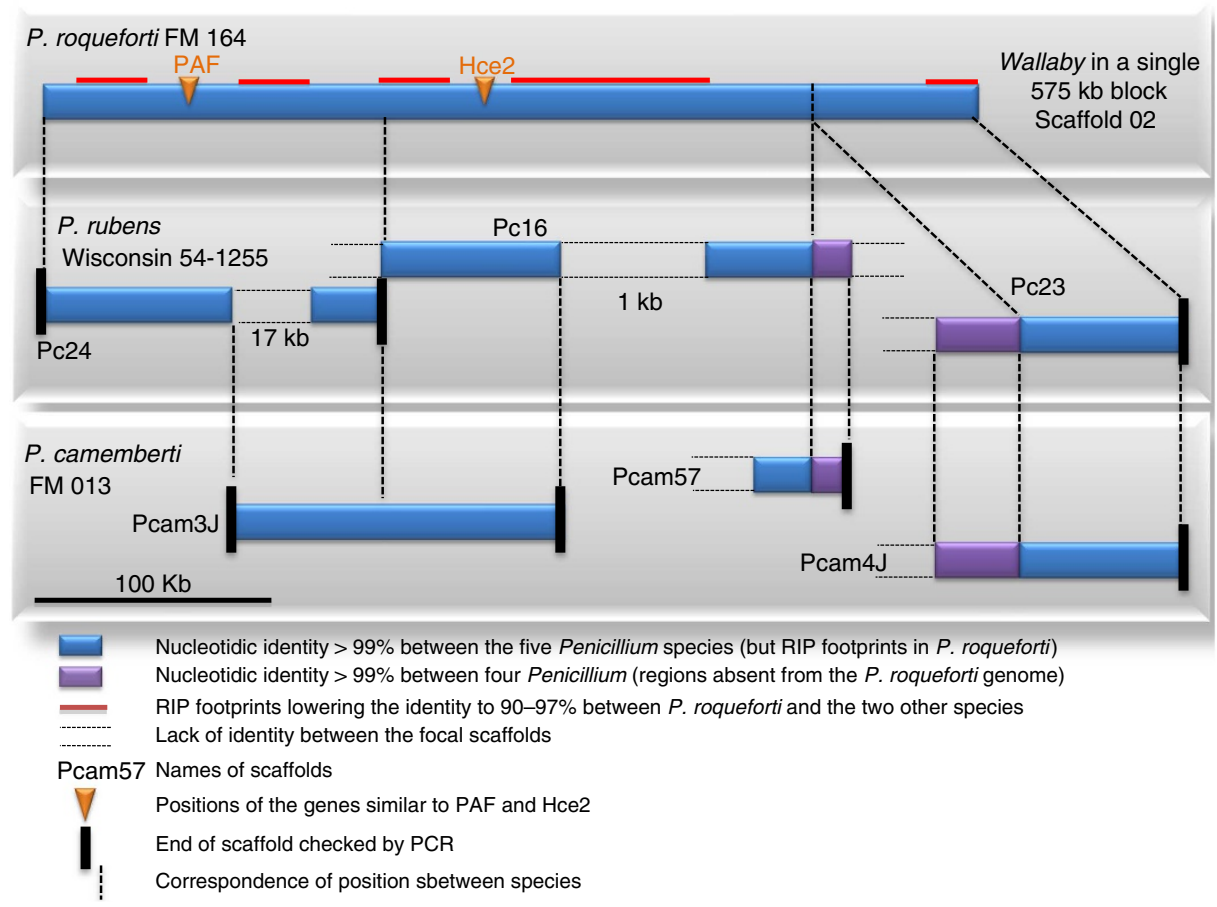

Figure 2 | Comparative genomic structure of Wallaby. The structure of the horizontally transferred genomic island is compared between $P$. roqueforti, $P$. rubens and $P$. camemberti. The sequence shown at the top corresponds to the Wallaby locus in P. roqueforti FM164, that is, from positions $1,487,035$ to $2,061,670$.
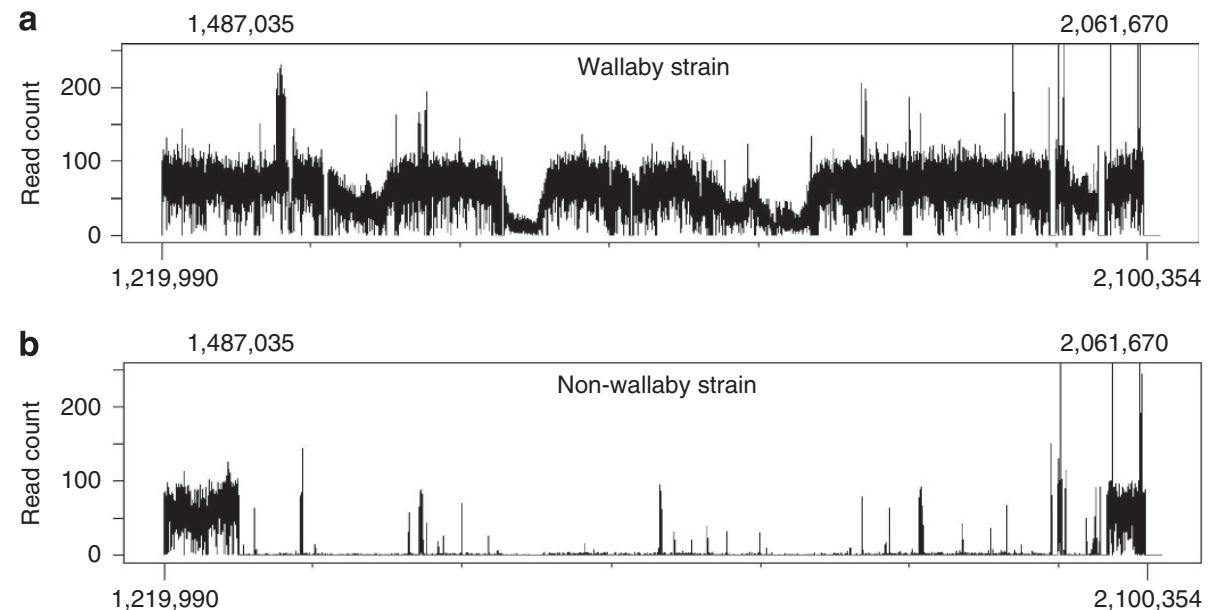

Figure 3 | Read mapping of the genomes of two additional strains against the Wallaby locus of the P. roqueforti FM164. (a) A strain bearing Wallaby isolated from cheese. (b) A strain not possessing Wallaby, isolated from an environment other than dairy products. This figure displays a count of the number of reads at each single base position. The $x$ axis represents genomic coordinates. The $y$ axis scale represents the number of reads counted for every position.

amplification with Wallaby-specific primers, designed from single-copy putative coding sequences, each amplifying $1 \mathrm{~kb}$ (Supplementary Table S1). We carried out an exhaustive screening of all the terverticillate Penicillium species, the clade to which $P$. roqueforti, $P$. camemberti and $P$. rubens belong, from the public collection of the MNHN, as well as subsets of additional collections, including strains isolated from various ecological niches, corresponding to 51 morphospecies (Fig. 5, Supplementary Tables S2-S4). Amplicons were obtained for all $P$. camemberti strains and a fraction of the strains from $P$. roqueforti and from the $P$. chrysogenum $-P$. rubens clade. The amplicons were sequenced and all were identical to Wallaby sequences. The lack of non-synonymous substitutions in all these species further indicates that the presence of Wallaby is not an ancestral character. Several strains from species closely related to $P$. camemberti and present in dairy environments or occurring as food contaminants, such as $P$. caseifulvum, $P$. biforme, $P$. fuscoglaucum, P. palitans, P. solitum, $P$. nordicum and P. polonicum, for example, also gave amplicons (Fig. 5; Supplementary Tables S2 and S3). No amplicons were obtained from $P$. roqueforti strains isolated from other environmental niches or from Penicillium species not associated with cheese environments (for example, $P$. carneum, $P$. expansum, $P$. cavernicola), other than $P$. chrysogenum s. $l$., that is, the species complex encompassing both P. chrysogenum and P. rubens (Fig. 5; Supplementary Tables S2 and S3). 
Table 2 | Pearson correlation coefficients for Z-score of tetranucleotide frequency.

\begin{tabular}{|c|c|c|c|c|c|c|c|c|c|c|}
\hline & Wallaby & $\begin{array}{l}\text { P. roqueforti } \\
\text { WoWallaby }\end{array}$ & $\begin{array}{l}\text { P. rubens } \\
\text { WoWallaby }\end{array}$ & $\begin{array}{l}\text { P. camemberti } \\
\text { WoWallaby }\end{array}$ & $\begin{array}{c}P . \\
\text { digitatum }\end{array}$ & $\begin{array}{c}P . \\
\text { carneum }\end{array}$ & $\begin{array}{c}\text { A. } \\
\text { flavus }\end{array}$ & $\underset{\text { kawachii }}{A .}$ & $\begin{array}{c}\text { A. } \\
\text { niger }\end{array}$ & $\begin{array}{c}\text { A. } \\
\text { fumigatus }\end{array}$ \\
\hline $\begin{array}{l}\text { P. roqueforti } \\
\text { WoWallaby }\end{array}$ & 0.917 & & & & & & & & & \\
\hline $\begin{array}{l}\text { P. camemberti } \\
\text { WoWallaby }\end{array}$ & 0.917 & 0.992 & 0.992 & & & & & & & \\
\hline A. flavus & 0.870 & 0.950 & 0.947 & 0.944 & 0.878 & 0.943 & & & & \\
\hline A. kawachii & 0.869 & 0.934 & 0.930 & 0.931 & 0.857 & 0.922 & 0.967 & & & \\
\hline A. niger & 0.848 & 0.930 & 0.922 & 0.922 & 0.860 & 0.923 & 0.970 & 0.995 & & \\
\hline A. fumigatus & 0.885 & 0.936 & 0.932 & 0.929 & 0.849 & 0.922 & 0.963 & 0.960 & 0.959 & \\
\hline A. oryzae & 0.872 & 0.950 & 0.948 & 0.945 & 0.880 & 0.943 & 1.000 & 0.967 & 0.969 & 0.963 \\
\hline
\end{tabular}

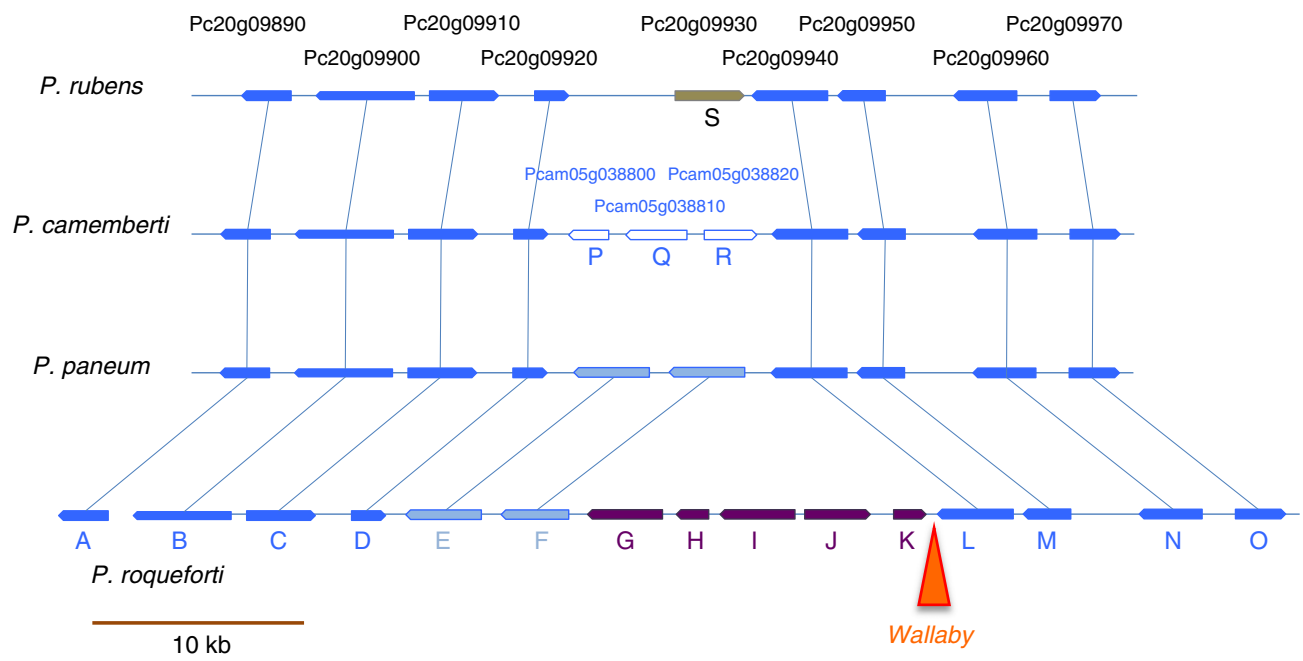

Figure 4 | Schematic representation of the flanking regions of Wallaby in P. roqueforti compared with homologous regions in other Penicillium species. Predicted genes are indicated, and those for which homology could be detected in this region in other species are linked and blue. White predicted genes have no detectable similarity to sequences in this region from other species. Wallaby in $P$. roqueforti is indicated by a red triangle. The $P$. camemberti and $P$. rubens Wallaby regions are fragmented and located in non-homologous regions and are therefore not shown on this figure. $P$. paneum does not carry Wallaby.

Transferred region is potentially involved in competition. In P. roqueforti, Wallaby was predicted to contain 248 genes, 149 of which were covered by expressed sequence tags. No expanded gene families were detected within Wallaby. Few genes could be annotated (Table 3 and see Supplementary Fig. S7 for the gene ontologies recovered) and Fisher's exact tests indicated no significant enrichment of any particular function. Interestingly, some of the annotated genes were predicted to be involved in the regulation of conidiation (spore production) or in antimicrobial activities, suggesting functional advantages for Wallaby-bearing strains associated with competition in the cheese, which contain many other microorganisms.

In particular, among expressed genes within Wallaby, afp (gene ID: ProqFM164S02.2755) encodes a protein identical to PAF (Penicillium antifungal protein), with $100 \%$ nucleotide sequence identity to the paf gene of $P$. rubens; PAF has been experimentally shown to be cytotoxic to fungi ${ }^{24}$ and to regulate spore production ${ }^{25}$. Another expressed protein with putative antimicrobial activities, ProqFM164S02g002866 (gene ID: ProqFM164S02.2870), matched to Hce $2^{26}$, which has an Ecp2 domain-originally identified as a virulence factor in Cladosporium fulvum-fused to a GH18 chitinase domain very similar to the $\alpha$ subunit of the yeast killer toxin zymocin from the dairy yeast Kluyveromyces lactis ${ }^{27}$. Hce 2 proteins with such an architecture are thought to play a role in antagonistic interactions with other microorganisms ${ }^{26}$.

\section{Discussion}

The sequencing of the first two genomes of food Penicillium strains will provide an invaluable resource for comparative genomics of fungi, nicely complementing the 1,000 fungal genomes project and the JGI fungal genome initiative, in which no Penicillium from the food environment is being sequenced. We also report here the development and application of a methodology for improving and validating genome assemblies based on an original single-DNA molecule technology, molecular combing. Improving genome assembly, notably by the use of physical maps, has been largely advocated ${ }^{28,29}$, and is even deemed crucial for comparative genomics ${ }^{30}$.

Our data indicate that food Penicillium strains contain regions, grouped at a single location and called Wallaby in P. roqueforti, 


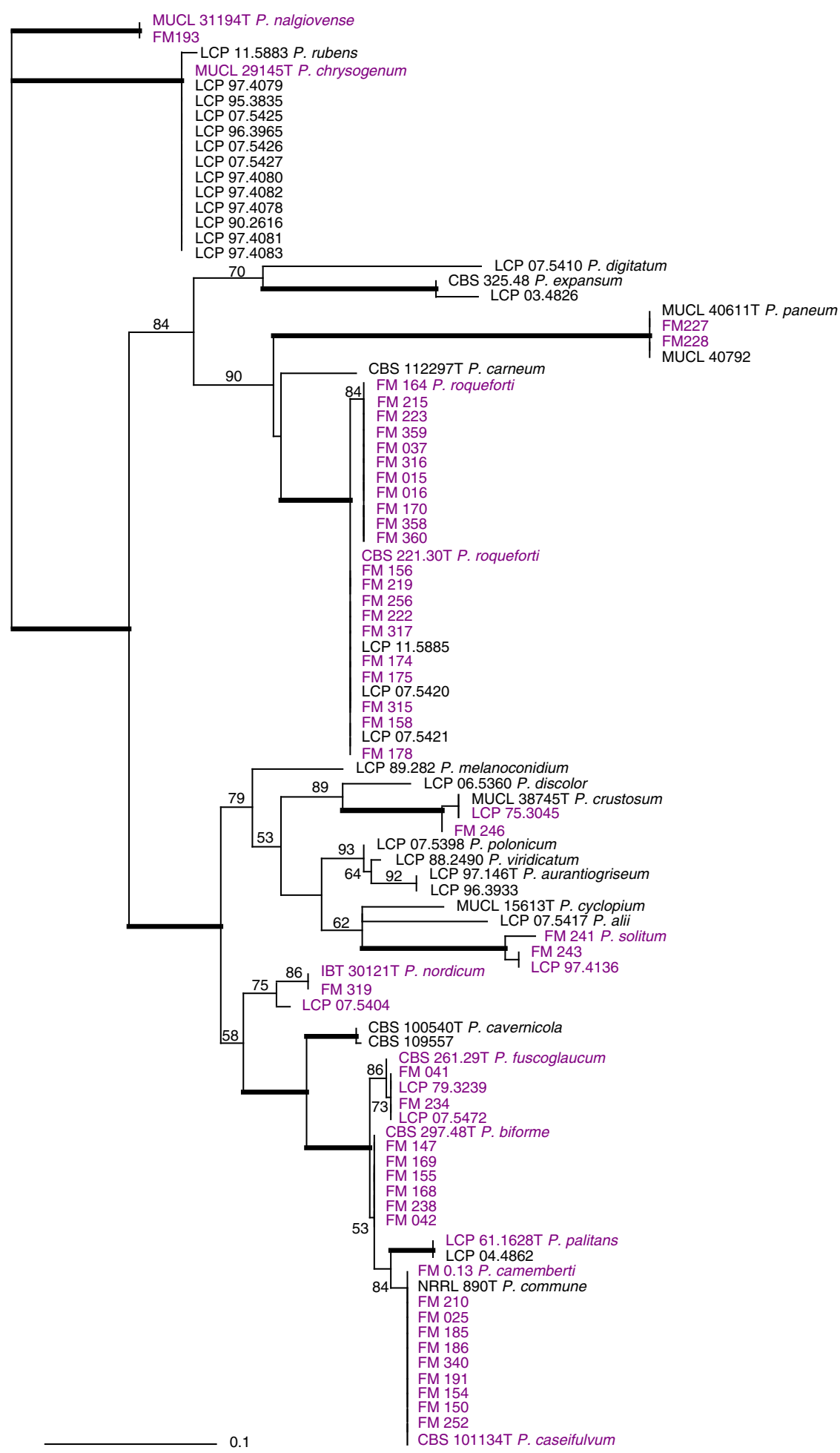

\begin{tabular}{|c|c|c|c|}
\hline \begin{tabular}{l|l}
$1 \mathrm{a}$ & 1 \\
\end{tabular} & \begin{tabular}{l|l}
$1 \mathrm{~b}$ & 2 \\
\end{tabular} & & \\
\hline & & - MUCL 31194T & $P$. nalgiovense \\
\hline & & LCP 11.5883 & $P$. rubens \\
\hline & & $\begin{array}{l}\text { MUCL 29145T } \\
\end{array}$ & \\
\hline & & LCP 95.3835 & \\
\hline & & $\begin{array}{l}\text { LCP } 07.5425 \\
\text { LCP } 96.3965\end{array}$ & \\
\hline & & $\begin{array}{l}\text { LCP } 07.5426 \\
\text { LeP } 07.5427\end{array}$ & P. chrysogenum \\
\hline & & L'Cp 97.4080 & \\
\hline & & LCP 97.4078 & \\
\hline & & 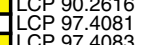 & \\
\hline
\end{tabular}
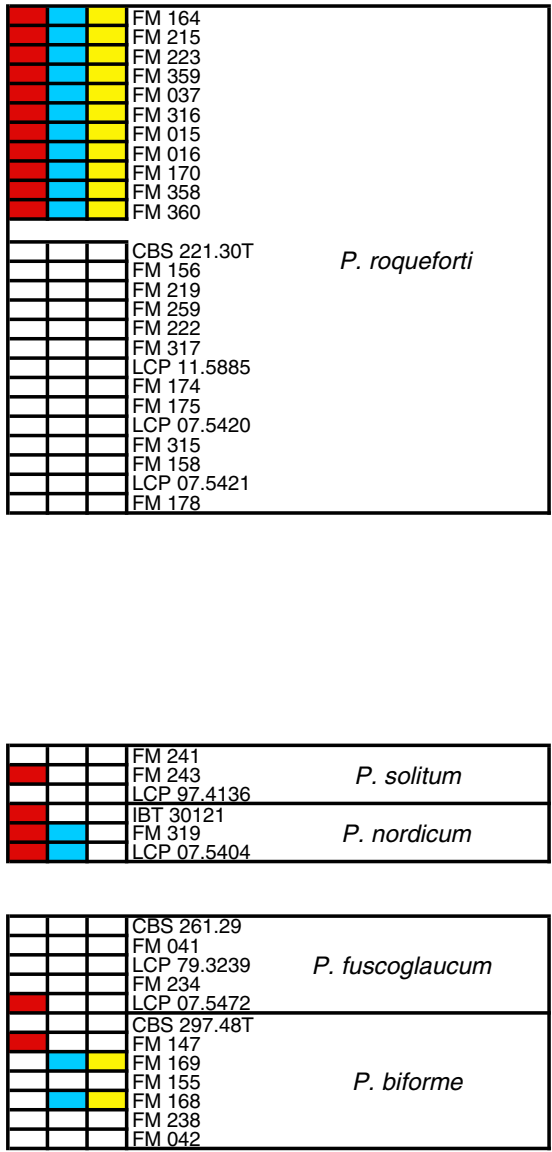

Strains isolated from dairy and considered by producers either as desired or as contaminate species

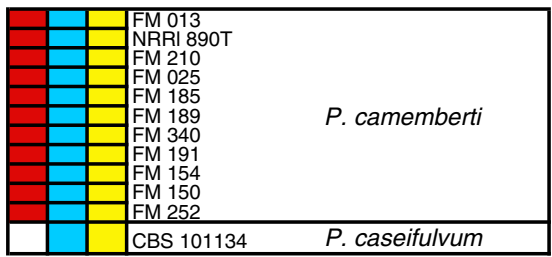

Strains isolated from other environments

Figure 5 | Gene genealogy for the Penicillium strains screened by PCR for the presence of Wallaby and for which DNA sequences of the markers could be obtained. Penicillium species associated with the cheese environment are indicated in purple. Colours in the pseudotable indicate positive amplification for the three primer pairs (red, blue and yellow, respectively) for single-copy predicted genes in Wallaby.

that have very recently undergone multiple horizontal transfers. Several lines of evidence support the acquisition of Wallaby by horizontal transfer and rule out alternative hypotheses. First, an ancestral presence of Wallaby is refuted by the complete identity (except for RIP mutation footprints in some fragments in $P$. roqueforti), even in noncoding regions, over more than $500 \mathrm{~kb}$, between distant species with genome sequence identities otherwise of about $90 \%$, and by the absence of Wallaby from all 


\section{Table 3 | Putative functions of the genes located in the flanking regions of Wallaby in $P$. roqueforti.}

\begin{tabular}{lcl} 
Genes & Protein length (aa) & \multicolumn{1}{c}{ InterPro annotation } \\
\hline A & 401 & Protein of unknown function \\
B & 757 & Autocrine motility factor receptor \\
C & 555 & Transporter protein \\
D & 236 & Hypothetical protein \\
E & 264 & Hypothetical protein \\
F & 176 & Hypothetical protein \\
G & 574 & Phosphate transporter \\
H & 133 & Helix-loop-helix domain \\
I & 564 & Carboxypeptidase \\
J & 768 & Oligopeptide transporter \\
K & 459 & Nucleotidyltransferase \\
L & 526 & Cytochrome P450 \\
M & 353 & Methyltransferase \\
N & 489 & Motif in proteasome subunits \\
O & 313 & Dehydrogenase \\
P & 336 & Alcohol dehydrogenase \\
Q & 606 & Unknown function \\
R & 558 & Cytochrome P450 \\
S & 480 & Oxydase \\
\hline
\end{tabular}

The letters correspond to those on the Fig. 4. The length of the genes is given in amino-acid numbers and the annotation is provided as obtained from the InterPro database.

fungal species other than those in which it is identical. Furthermore, the non-homologous locations rule out introgression and the presence of Wallaby almost exclusively in species from the food environment and the different nucleotide composition of Wallaby from the rest of the recipient genomes provide further support for horizontal transfer. The nucleotide composition of Wallaby suggests that the donor species may belong to the Aspergillus or Penicillium clade. The function of one of the genes contained in Wallaby, experimentally demonstrated in $P$. rubens ${ }^{24}$, suggests that it may confer adaptation associated with competition with other microorganisms.

The occurrence of gene transfers in fungi has been reported before ${ }^{1,11,31,32}$, but this case of HGT is exceptional in several ways. The Wallaby genomic islands are unusually large, covering $2 \%$ of the host genomes. Furthermore, these sequences were found to be $100 \%$ identical in the species screened, with the exception of the RIP mutation footprints in $P$. roqueforti. The complete identity of the transferred sequences and the lack of corresponding sequences in databases prevent phylogenetic reconstruction of the history of transfers. However, we can hypothesize that the original donor had a complete island in a single block, as in $P$. roqueforti, and that this block was then transferred to other acceptor genomes, in which it was fragmented and may then have been transferred to other species. Some of the $P$. rubens scaffolds containing Wallaby, Pc23 and Pc24 have already been shown to be absent from P. digitatum ${ }^{19}$. This study also showed that several fragments of these scaffolds matched other dispersed elements in the genome of $P$. rubens, with an identity of about $90 \%$. It has been suggested that this level of similarity indicated gene family expansion rather than horizontal transfer ${ }^{19}$. Our analysis of additional sequenced genomes revealed that these dispersed elements were specific to $P$. rubens and that horizontal transfers occurred in addition to possible gene family expansions.

The widespread occurrence of Wallaby in cheese species, including species never found in other environments, such as $P$. camemberti, together with the identity of the sequences indicate that these transfers occurred in cheese and were, therefore, very recently promoted by human activities. Such horizontal transfers may be facilitated by the ability of fungi to form anastomoses-somatic fusions between mycelia known to occur in Penicillium species 33,34 - and may facilitate the transfer of whole chromosomes in fungi grown in laboratory environment ${ }^{11}$. Functions brought by the transferred material may, therefore, be beneficial in the food environment, as suggested by the functions identified in Wallaby, involved in antagonistic interactions with other microorganisms ${ }^{26}$.

This study provides strong support for the view that horizontal transfers of large genomic regions play an important role in fungal adaptation to new environments ${ }^{35,36}$, including those created by humans. The example reported here is, however, unique to date among transfers, in terms of the size of the region transferred, its total identity between species and the number of species involved. Horizontal gene transfer in fungi may be beneficial, particularly in cheese making strains or wine yeasts, in which large transfers of genes promoting fermentation have been detected $^{1}$. The data presented here, particularly when considered in the light of the growing list of reports of horizontal gene transfer between eukaryotes, suggest that this phenomenon may occur frequently and may have a wider impact than previously thought, as already advocated ${ }^{3,5}$. We foresee major implications for the management of pathogen species in the face of current changes, including globalization, which may bring previously isolated species into contact. Rapid evolution by horizontal gene transfer may also have a major impact through the emergence of new diseases caused by fungi acquiring new virulence genes horizontally 7,10,11,15,37. Furthermore, in itself, this finding indicates that horizontal gene transfer can occur within the food chain. The frequency of this phenomenon should be investigated more thoroughly, as it may have a potential impact on food, agricultural and biotechnological practices.

\section{Methods}

DNA extraction and genome sequencing. DNA was extracted with the cetyltrimethylammonium bromide (CTAB) extraction procedure ${ }^{38-40}$. Mycelium (10 g) was recovered from $\sim 10$ Petri dishes and frozen with liquid nitrogen in a mortar. The mycelium was ground to a powder and $10 \mathrm{ml}$ of $2 \times$ CTAB buffer was added. The frozen mixture was left to thaw, quickly transferred to a $50 \mathrm{ml}$ Corning tube and placed in a $65^{\circ} \mathrm{C}$ water bath for $30 \mathrm{~min}$. One volume of chloroform/isoamyl alcohol was then added and the solution was mixed thoroughly to form a complete emulsion. After a centrifugation step of $10 \mathrm{~min}$ at $4,000 \mathrm{~g}$, the top aqueous phase (above the chloroform phase and mycelium fragments) was transferred into a new $50 \mathrm{ml}$ tube; $1 / 5$ volume of $5 \%$ CTAB solution was added and the chloroform/ isoamyl alcohol extraction step was performed again, except for the addition of the $5 \%$ CTAB solution at the end. The top aqueous phase was gently mixed with an equal volume of $\mathrm{CTAB}$ precipitation buffer and the resulting solution was incubated for $1 \mathrm{~h}$ at room temperature to form a precipitate. After $10 \mathrm{~min}$ of centrifugation at $4,000 \mathrm{~g}$, the supernatant was discarded and the precipitate dissolved into $2 \mathrm{ml}$ of high-salt TE ( $\mathrm{pH} 8.0$ ) [10 mM tris(hydroxymethyl)aminomethane (TRIS), $1 \mathrm{mM}$ ethylenediaminetetraacetic acid (EDTA) and $1 \mathrm{M} \mathrm{NaCl}$. Nucleic acids were precipitated with $4 \mathrm{ml}$ of cold ethanol, pelleted in Corex tubes and washed with $4 \mathrm{ml}$ of $70 \%$ ethanol. The nucleic acid pellet was left to dry. The pellet was resuspended into $4 \mathrm{ml}$ of TE ( $\mathrm{pH}$ 8.0) (10 mM TRIS, $1 \mathrm{mM}$ EDTA). Nuclear DNA was purified and excess mitochondrial DNA was eliminated by centrifugation on a caesium gradient with $4^{\prime}, 6$-diamidino-2-phenylindole (DAPI) ${ }^{41}$. We added $1.15 \mathrm{~g}$ solid caesium chloride and $4 \mu \mathrm{l}$ of DAPI solution $\left(1 \mathrm{mg} \mathrm{ml}^{-1}\right)$ per $\mathrm{ml}$ of DNA solution (final density: $1.65 \mathrm{~g} \mathrm{ml}^{-1}$ ). The DNA/CsCl/DAPI solution was centrifuged in a Quick-Seal centrifuge tube (part \#342412, Beckman, Palo Alto, USA) at 50,000 r.p.m., for $12-15$ h, in a Beckman VTi65 vertical rotor. Two bands of DNA were visible under UV light. The lower band (that is, nuclear DNA, the upper band corresponding to mitochondrial DNA) was collected with a syringe $(1.2 \times 40 \mathrm{~mm}$ needle); DAPI was extracted with isoamyl alcohol saturated with $\mathrm{CsCl}\left(1.15 \mathrm{~g} \mathrm{ml}^{-1}\right)$ and the DNA solution was dialyzed for 4 days against $10 \mathrm{mM}$ TRIS, $1 \mathrm{mM}$ EDTA ( $\mathrm{pH} 8.0$ ) with twice-daily replacement of the dialysis solution.

Sequencing and annotation. Penicillium camemberti FM 013 and P. roquefort $i$ FM164 were sequenced by Genoscope (Evry, France), via the 454 sequencing of an $8 \mathrm{~kb}$ mate pair library (793786 and 661034 cleaned read pairs, respectively) combined with Illumina Solexa sequencing (34126183 and 3425303176 nt single-end reads, respectively). Assembly was performed with SOAPdenovo v1.05 and velvet v1.1.04 (ref. 42). SOAPdenovo was run (kmer values 43-67) to generate contigs. Velvet was then run with combined raw reads and SOAPdenovo contigs (parameters '-cov_cutoff 5 -min_contig_lgth 100 -max_divergence 0.05 - 
long_mult_cutoff 1 -exp_cov auto'). The range of kmer values for Velvet was 4157. The short-reads assembly with the maximum N50 value was used as input for the scaffolding process (SOAPprepare and SOAPdenovo). For P. roqueforti, maximum $\mathrm{N} 50$ values were obtained with a kmer value of 37 and a minimum of six links between contigs (parameter (default $=5$ ) pair_num_cutoff $=6$ ). For P. camemberti, a kmer value of 27 and a minimum of 14 links gave the highest N50 value.

Gene models were predicted with EuGene ${ }^{43,44}$, a highly integrative eukaryotic protein-coding gene prediction platform. This gene predictor requires training: a data set of 442 curated gene models was built and split into three homogeneous, independent data sets. The first was used for training, the second for parameter optimization and the third for performance evaluation. We used for genome annotation 13,878 ESTs, resulting from the assembly of 780,051 cDNA sequences from P. roqueforti, together with the Swiss Protein database (February 2011), the proteomes of Saccharomyces cerevisiae and P. chrysogenum Wisconsin 54-1255, a database of all Eurotiales proteins in GenBank HG765102-HG778979 (February 2011). Transposable elements were identified with REPET ${ }^{45}$. InterPro was used to identify protein domains and families.

The P. roqueforti FM164 draft genome was assembled before molecular combing into 73 scaffolds of over $2 \mathrm{~kb}$, spanning $28 \mathrm{Mb}$. The P. camemberti FM 013 draft genome spans $34 \mathrm{Mb}$, assembled into 140 scaffolds of over $2 \mathrm{~kb}$.

Fungal isolates and single-spore isolation. We used 441 isolates in total (Supplementary Tables S2-S4). The 124 isolates provided by producers of starter cultures and cheeses were labelled FM and their origins are confidential. All were isolated from the cheese environment and belong to four distinct ascomycete genera: Penicillium, Fusarium, Scopulariopsis and Sporendonema ${ }^{46}$. We analysed all 241 terverticillate Penicillium strains from the public collection of the MNHN, and some Eupenicillium species. We also analysed the LUBEM-Brest collection encompassing $76 P$. roqueforti isolates, labelled with an F. These isolates were obtained from blue cheeses from 14 countries. The numbers after the ' $F$ ' correspond to individual cheeses. For the 39 cheeses, morphologically different strains were treated as different strains, labelled by a number following that identifying the cheese (for example, F17.1 and F17.2 are two strains from cheese 17). Single-spore isolation was systematically performed by the dilution method, after growth for 3-5 days at $25^{\circ} \mathrm{C}$ on malt agar. The ' $\mathrm{F}$ ' strains were also obtained by spore dilution.

DNA extraction for PCR amplification. Genomic DNA was extracted from fresh mycelium of the isolates listed in Supplementary Tables S2-S4. Mycelium was obtained after 3-5 days on malt agar for Penicillium, Scopulariopsis and Fusarium strains and on a confidential medium provided by starter producers for Sporendonema casei. The Qiagen DNeasy Plant Mini Kit (Qiagen Crawley, UK) was used for DNA extraction.

PCR amplification and sequencing of amplicons. PCR was performed in a volume of $50 \mu \mathrm{l}$, containing $25 \mu \mathrm{l}$ template DNA, $1.25 \mathrm{U}$ AmpliTaq DNA polymerase (Roche Molecular Systems, Branchburg, NJ, USA), $5 \mu 110 \times$ Taq DNA polymerase buffer, $5 \mu \mathrm{l} 50 \%$ glycerol, $2 \mu \mathrm{l} 5 \mathrm{mM}$ dNTPs, $2 \mu \mathrm{l}$ of each $10 \mu \mathrm{M}$ primer and $50-100 \mathrm{ng}$ template DNA. Strains were identified with the $5^{\prime}$ end of the $\beta$-tubulin gene, with primers $\mathrm{Bt} 2 \mathrm{a} / \mathrm{Bt} 2 \mathrm{~b}$ (ref. 47 ). The three primer pairs designed to detect Wallaby are shown in Supplementary Table S1. DNA fragments PC4 and PC13 (ref. 48) were used for the phylogenetic analysis. Amplifications were performed with 30 cycles of $30 \mathrm{~s}$ at $95^{\circ} \mathrm{C}, 30 \mathrm{~s}$ at $58^{\circ} \mathrm{C}$ for the three Wallaby primers, amplifying $1 \mathrm{~kb}$ each, and $2 \mathrm{~min}$ at $72^{\circ} \mathrm{C}$. For microsatellite loci, the thermal regime was 35 cycles of $30 \mathrm{~s}$ at $94^{\circ} \mathrm{C}, 30 \mathrm{~s}$ at $50{ }^{\circ} \mathrm{C}$ and $30 \mathrm{~s}$ at $72^{\circ} \mathrm{C}$. All PCR programs had a final 5 min extension step at $72^{\circ} \mathrm{C}$. PCR products were purified and sequenced by Genoscope (Évry, France).

Protoplast preparation for molecular combing. A $P$. roqueforti conidial suspension was plated on $\mathrm{M} 3$ medium $\left(0.25 \mathrm{gl}^{-1} \mathrm{KH}_{2} \mathrm{PO}_{4}, 0.3 \mathrm{gl}^{-1} \mathrm{~K}_{2} \mathrm{HPO}_{4}\right.$, $0.25 \mathrm{gl}^{-1} \mathrm{MgSO}_{4}, 0.5 \mathrm{gl}^{-1}$ urea, $0.05 \mathrm{mgl}^{-1}$ thiamine, $0.25 \mathrm{\mu gl}^{-1}$ biotin, $2.5 \mathrm{mgl}^{-1}$ citric acid, $2.5 \mathrm{mgl}^{-1} \mathrm{ZnSO}_{4}, 0.5 \mathrm{mgl}^{-1} \mathrm{CuSO}_{4}, 125 \mu \mathrm{gl}^{-1} \mathrm{MnSO}_{4}$, $25 \mathrm{gl}^{-1}$ boric acid, $25 \mathrm{gl}^{-1}$ sodium molybdate, $25 \mu \mathrm{gl}^{-1}$ iron alum, $5 \mathrm{gl}^{-1}$ glucose, $25 \mathrm{mgl}^{-1}$ chloramphenicol in seven Petri dishes and incubated 4 days at $25^{\circ} \mathrm{C}$. Conidia were harvested and resuspended in $20 \mathrm{ml} \mathrm{M} 3$ medium. Two $50 \mathrm{ml}$ M3 medium liquid cultures were inoculated with the conidial suspension. Germination occurred after $18 \mathrm{~h}$ of incubation at $25^{\circ} \mathrm{C}$, with shaking at 90 r.p.m. The germinating conidia were harvested by centrifugation $(10 \mathrm{~min}, 2,640 \mathrm{~g})$. Dried mycelium ( $1 \mathrm{~g}$ ) was suspended in the protoplast isolation solution $(400 \mathrm{mg}$ Filtrozym (Laffort), $20 \mathrm{mg}$ bovine serum albumin in $10 \mathrm{ml}$ of TRF1 solution (1.2 $\mathrm{M} \mathrm{MgSO}_{4}, 10 \mathrm{mM}$ orthophosphate, $\mathrm{pH}$ 5.8). The suspension was incubated at $30{ }^{\circ} \mathrm{C}$ for $120-150 \mathrm{~min}$, with shaking at 90 r.p.m. Once the cell walls had been lysed, we transferred the $10 \mathrm{ml}$ suspension to a $30 \mathrm{ml}$ glass tube and overlaid it with $10 \mathrm{ml}$ TRF2 (0.6 M sorbitol, $100 \mathrm{mM}$ Tris- $\mathrm{HCl} \mathrm{pH} \mathrm{7.5).} \mathrm{After} \mathrm{centrifugation} \mathrm{for} 10 \mathrm{~min}$ at $2869 \mathrm{~g}$ at room temperature, the protoplasts formed a layer at the interface. The protoplast layer was removed and washed with an equivalent volume of TRF3

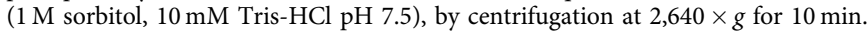
The protoplast pellet was suspended in $1 \mathrm{ml}$ TRF4 solution (1 M sorbitol, $10 \mathrm{mM}$ Tris- $\mathrm{HCl} \mathrm{pH} \mathrm{7.5,} 10 \mathrm{mM} \mathrm{CaCl}_{2}$ ).
Molecular combing. Molecular combing and suitable hybridization, detection and scanning procedures were performed according to conventional combing procedures $^{49,50}$. Molecular combing experiments involve embedding cells into an agarose plug, releasing DNA into solution by digesting this plug, stretching the DNA on a coverslip with a molecular combing system and performing fluorescent in situ hybridization experiments on the combed DNA. Briefly, P. roqueforti FM164 protoplast prepared as described above were embedded in agarose plugs. Protoplasts were lysed overnight by incubation in a $1 \%$ sarkosyl, proteinase $\mathrm{K}$ $\left(2 \mathrm{mg} \mathrm{ml}^{-1}\right)$ in $0.5 \mathrm{M}$ EDTA pH 8.0 at $50^{\circ} \mathrm{C}$. Agarose plugs were then washed in $10 \mathrm{mM}$ Tris, $1 \mathrm{mM}$ EDTA pH 8.0. Genomic DNA was stained by incubating the plug in a $40 \mathrm{mM}$ Tris, $2 \mathrm{mM}$ EDTA, $3 \mu \mathrm{l}$ YoYo- 1 solution for $1 \mathrm{~h}$. The combing solution containing the genomic DNA was obtained by melting agarose at $68^{\circ} \mathrm{C}$ for $20 \mathrm{~min}$ and subsequently digesting agarose molecules using $\beta$-agarase $\left(42^{\circ} \mathrm{C}\right.$, overnight). DNA fibres were combed on combicoverslips using the molecular combing system (both from Genomic Vision, France). The PCR amplicons for probe production were purified and used directly as templates for the labelling reaction, rather than after subcloning (primer sequences available on request). Probes were labelled by random priming and revealed using Alexa 594 (red), Alexa 350/AMCA (blue) conjugated antibody sandwiches. The combed DNA was counterstained after the fluorochrome detection step, by incubating the coverslip in $30 \mu \mathrm{l}$ of a 1:1,000 YoYo-1 solution in milli-Q water for $30 \mathrm{~s}$ and then washing three times, for three minutes each, in milli-Q water.

Genomic Morse codes (GMC) were designed at the extremities of scaffolds. A GMC is a signal generated by a set of specifically designed probes. A GMC consists of an alternating series of dots, dashes (probes) and gaps (region between two probes) of different sizes and colours, designed to generate an unambiguous pattern at a specific locus, thus physically mapping the region ${ }^{51}$. GMCs at the extremities of scaffolds generate unambiguous patterns making it possible to locate and orient scaffolds accurately on single-stretch DNA molecules, by combining two Morse codes in cases of neighbouring scaffolds. In the case of distant scaffolds, the distance separating the scaffolds is measured based on the distance separating the two genomic Morse codes (Supplementary Fig. S2).

Phylogenetic analysis. Genealogical relationships were inferred using the flanking regions of the two microsatellite loci PC4 and PC13 (classical loci like the $\beta$-tubulin gene are not variable enough). Sequences were manually aligned, with BioEdit ${ }^{52}$. Independent phylogenetic trees were built with TreeFinder ${ }^{53}$ under a maximum likelihood framework. A nucleotide substitution model $(\mathrm{GTR}+\mathrm{G})$ was inferred with jmodeltest ${ }^{54}$. As the two topologies were congruent, we concatenated the data sets to obtain a single phylogenetic tree based on $363 \mathrm{bp}$. Branch support was determined from a bootstrap analysis of 1,000 re-sampled data sets.

RIP mutation analysis. Using fragments of the Wallaby sequences of $P$. roqueforti FM164, $P$. camemberti FM 013 and $P$. rubens Wisconsin 54-1,255, we searched for RIP-like footprints ${ }^{55}$. Multiple sequence alignments were built, using ClustalW with default settings ${ }^{56}$. RIP mutation-like footprints were sought with RIPCAL software $^{57}$ (Supplementary Fig. S6).

Tetranucleotide composition. Correlations between $Z$-scores of tetranucleotide composition were assessed using jspecies version 1.2.1 ${ }^{61}$.

\section{References}

1. Novo, M. et al. Eukaryote-to-eukaryote gene transfer events revealed by the genome sequence of the wine yeast Saccharomyces cerevisiae EC1118. Proc. Natl Acad. Sci. USA 106, 16333-16338 (2009).

2. Gibbons, J. G. et al. The evolutionary imprint of domestication on genome variation and function of the filamentous fungus Aspergillus oryzae. Curr. Biol. 22, 1403-1409 (2012).

3. Keeling, P. J. Functional and ecological impacts of horizontal gene transfer in eukaryotes. Curr. Opin. Genet. Dev. 19, 613-619 (2009).

4. Andersson, J. O. Gene transfer and diversification of microbial eukaryotes. Annu. Rev. Microbiol. 63, 177-193 (2009).

5. Syvanen, M. Evolutionary implications of horizontal gene transfer. Annu. Rev. Genet. 46, 341-358 (2012).

6. Alsmark, C. et al. Patterns of prokaryotic lateral gene transfers affecting parasitic microbial eukaryotes. Genome Biol. 14, R19 (2013).

7. Friesen, T. L. et al. Emergence of a new disease as a result of interspecific virulence gene transfer. Nat. Genet. 38, 953-956 (2006).

8. Temporini, E. D. \& VanEtten, H. D. An analysis of the phylogenetic distribution of the pea pathogenicity genes of Nectria haematococca MPVI supports the hypothesis of their origin by horizontal transfer and uncovers a potentially new pathogen of garden pea: Neocosmospora boniensis. Curr. Genet 46, 29-36 (2004).

9. Mehrabi, R. et al. Horizontal gene and chromosome transfer in plant pathogenic fungi affecting host range. FEMS Microbiol. Rev. 35, 542-554 (2011).

10. Slot, J. C. \& Rokas, A. Horizontal transfer of a large and highly toxic secondary metabolic gene cluster between fungi. Curr. Biol. 21, 134-139 (2011). 
11. Ma, L.-J. et al. Comparative genomics reveals mobile pathogenicity chromosomes in Fusarium. Nature 464, 367-373 (2010).

12. Dunning Hotopp, J. C. et al. Widespread lateral gene transfer from intracellular bacteria to multicellular eukaryotes. Science 317, 1753-1756 (2007).

13. Yue, J., Hu, X., Sun, H., Yang, Y. \& Huang, J. Widespread impact of horizontal gene transfer on plant colonization of land. Nat. Commun. 3, 1152 (2012).

14. Altincicek, B., Kovacs, J. L. \& Gerardo, N. M. Horizontally transferred fungal carotenoid genes in the two-spotted spider mite Tetranychus urticae. Biol. Lett. 8, 253-257 (2012)

15. Sanders, I. R. Rapid disease emergence through horizontal gene transfer between eukaryotes. Trends Ecol. Evol. 21, 656-658 (2006).

16. Samson, R. A. Introduction to food-and airborne fungi. pp 389, (2000).

17. Pitt, J. I. \& Hocking, A. D. Fungi and Food Spoilage. Fungi food spoilage. pp 519 (Springer, 2009).

18. Van den Berg, M. et al. Genome sequencing and analysis of the filamentous fungus Penicillium chrysogenum. Nat. Biotechnol. 26, 1161-1168 (2008).

19. Marcet-Houben, M. et al. Genome sequence of the necrotrophic fungus Penicillium digitatum, the main postharvest pathogen of citrus. BMC Genomics 13, 646 (2012).

20. Conti, C. \& Bensimon, A. A combinatorial approach for fast, high-resolution mapping. Genomics 80, 135-137 (2002).

21. Lebofsky, R. \& Bensimon, A. Single DNA molecule analysis: applications of molecular combing. Brief. Funct. Genomic. Proteomic. 1, 385-396 (2003).

22. Braumann, I., van den Berg, M. \& Kempken, F. Repeat induced point mutation in two asexual fungi, Aspergillus niger and Penicillium chrysogenum. Curr. Genet. 53, 287-297 (2008).

23. Ropars, J. et al. Sex in cheese: evidence for sexuality in the fungus Penicillium roqueforti. PLoS One 7, e49665 (2012).

24. Kaiserer, L. et al. Characterization of the Penicillium chrysogenum antifungal protein PAF. Arch. Microbiol. 180, 204-210 (2003).

25. Hegedüs, N., Sigl, C., Zadra, I., Pócsi, I. \& Marx, F. The paf gene product modulates asexual development in Penicillium chrysogenum. J. Basic Microbiol. 51, 253-262 (2011).

26. Stergiopoulos, I. et al. In silico characterization and molecular evolutionary analysis of a novel superfamily of fungal effector proteins. Mol. Biol. Evol. 29, 3371-3384 (2012).

27. Jablonowski, D. \& Schaffrath, R. Zymocin, a composite chitinase and tRNase killer toxin from yeast. Biochem. Soc. Trans. 35, 1533-1537 (2007).

28. Lewin, H. A., Larkin, D. M., Pontius, J. \& O’Brien, S. J. Every genome sequence needs a good map. Genome Res. 19, 1925-1928 (2009).

29. Ricker, N., Qian, H. \& Fulthorpe, R. R. The limitations of draft assemblies for understanding prokaryotic adaptation and evolution. Genomics 100, 167-175 (2012).

30. Alkan, C., Sajjadian, S. \& Eichler, E. E. Limitations of next-generation genome sequence assembly. Nat. Methods 8, 61-65 (2011).

31. Rosewich, U. L. \& Kistler, H. C. Role of horizontal gene transfer in the evolution of fungi. Annu. Rev. Phytopathol. 38, 325-363 (2000).

32. Fitzpatrick, D. A. Horizontal gene transfer in fungi. FEMS Microbiol. Lett. 329, 1-8 (2012).

33. Haas, F. L., Lein, J., Moses, A. J. \& Puglisi, T. A. Heterokaryosis as a cause of culture rundown in Penicillium. Appl. Microbiol. 4, 187-195 (1956).

34. Gabriela Roca, M., Read, N. D. \& Wheals, A. E. Conidial anastomosis tubes in filamentous fungi. FEMS Microbiol. Lett. 249, 191-198 (2005).

35. Neafsey, D. E. et al. Population genomic sequencing of Coccidioides fungi reveals recent hybridization and transposon control. Genome Res. 20, 938-946 (2010).

36. Ellison, C. E. et al. Population genomics and local adaptation in wild isolates of a model microbial eukaryote. Proc. Natl Acad. Sci. USA 108, 1-10 (2011).

37. Slot, J. C. \& Hibbett, D. S. Horizontal transfer of a nitrate assimilation gene cluster and ecological transitions in fungi: a phylogenetic study. PLoS One 2 , e1097 (2007).

38. Rogers, S. \& Bendich, A. Extraction of DNA from milligram amounts of fresh, herbarium and mummified plant tissues. Plant Mol. Biol. 5, 69-76 (1985).

39. Rogers, S. \& Bendich, A. in Plant Molecular Biology Manual. (eds Gelvin, S. \& Schilperoot, R.) A6, 1-10 (Kluwer Academic Publishers, 1988).

40. Rogers, S. \& Bendich, A. in Plant Molecular Biology Manual. (eds Gelvin, S. \& Schilperoort, R. D1, 1-8 (Kluwer Academic Publishers, 1994).

41. Kapuscinski, J. DAPI: a DNA-specific fluorescent probe. Biotech. Histochem. 70, 220-233 (1995)

42. Zerbino, D. R. \& Birney, E. Velvet: algorithms for de novo short read assembly using de Bruijn graphs. Genome Res. 18, 821-829 (2008).

43. Schiex, T., Moisan, A. \& Rouzé, P. EuGène: an eukaryotic gene finder that combines several sources of evidence. Lect. Notes Comput. Sci. 2066, 111-125 (2001)
44. Foissac, S. et al. Genome annotation in plants and fungi: EuGène as a model platform. Curr. Bioinform. 3, 87-97 (2008).

45. Quesneville, H. et al. Combined evidence annotation of transposable elements in genome sequences. PLoS Comput. Biol. 1, 166-175 (2005)

46. Ropars, J., Cruaud, C., Lacoste, S. \& Dupont, J. A taxonomic and ecological overview of cheese fungi. Int. J. Food Microbiol. 155, 199-210 (2012).

47. Glass, N. \& Donaldson, G. Development of primer sets designed for use with the PCR to amplify conserved genes from filamentous ascomycetes. Appl. Environ. Microbiol. 61, 1323-1330 (1995).

48. Giraud, F. et al. Microsatellite loci to recognize species for the cheese starter and contaminating strains associated with cheese manufacturing. Int. J. Food Microbiol. 137, 204-213 (2010).

49. Michalet, X. et al. Dynamic molecular combing: stretching the whole human genome for high-resolution studies. Science 277, 1518-1523 (1997).

50. Cheeseman, K. et al. A diagnostic genetic test for the physical mapping of germline rearrangements in the susceptibility breast cancer genes BRCA1 and BRCA2. Hum. Mutat. 33, 998-1009 (2012).

51. Lebofsky, R., Heilig, R., Sonnleitner, M., Weissenbach, J. \& Bensimon, A. DNA replication origin interference increases the spacing between initiation events in human cells. Mol. Biol. Cell 17, 5337-5345 (2006).

52. Hall, T. BioEdit: a user-friendly biological sequence alignment editor and analysis program for Windows 95/98/NT. Nucl. Acids. Symp. Ser. 41, 95-98 (1999).

53. Jobb, G., von Haeseler, A. \& Strimmer, K TREEFINDER: a powerful graphical analysis environment for molecular phylogenetics. BMC Evol. Biol. 4, 18 (2004).

54. Posada, D. jModelTest 0.1.1. Syst. Biol. 1, 1-23 (2008).

55. Galagan, J. E. \& Selker, E. U. RIP: the evolutionary cost of genome defense. Trends Genet. 20, 417-423 (2004).

56. Thompson, J. D., Gibson, T. J. \& Higgins, D. G in Curr. Protoc. Bioinforma. (John Wiley \& Sons, Inc., 2002).

57. Hane, J. K. \& Oliver, R. P. RIPCAL: a tool for alignment-based analysis of repeat-induced point mutations in fungal genomic sequences. $B M C$ Bioinformatics 9, 478 (2008).

\section{Acknowledgements}

This work was supported by the ANR grant 'Food Microbiomes' (ANR-08-ALIA-007-02) coordinated by P.R. Molecular combing experiments were supported by Genomic Vision. K.C. and J.R. received joint CIFRE grants from ANRT and Genomic Vision, and ANRT and SPPAIL (Syndicat Professionnel des Producteurs d'Auxiliaires pour l'Industrie Laitière), respectively. A.B. received an ANR FROMA-GEN grant ANR-12-PDOC-0030. T.G. received an ERC starting grant, GenomeFun 309403. We thank the LUBEM laboratory of Brest (in particular, Emmanuel Coton and Monika Coton) for permission to use their strains (Supplementary Table S4). We thank Ricardo Rodriguez de la Vega for invaluable help with the genomic analyses. We thank Marco van den Berg for sharing raw data of the Penicillium chrysogenum Wisconsin 54-1255 strain (recently renamed Prubens).

\section{Author contributions}

P.R., Y.B. and J.D. designed the study. J.R. and K.C. performed the experiments. J.R., K.C., P.R., Y.B., A.Br., T.G., J.G., and E.S. analysed the data. T.G., J.R. and K.C. wrote the manuscript. Y.B., P.R., J.D. and F.M. revised the manuscript. A.Be., M.C., E.C., A.G., P.S., R.D., S.L., F.M., J.D. and A.-L.A. helped with experiments and/or provided strains or data. P.R., J.D., Y.B. and T.G. contributed to the funding of the study.

\section{Additional information}

Accession codes: Genome sequence data for P. roqueforti have been deposited in the GenBank nucleotide core database under the accession codes HG792015 to HG792062. Genome sequence data for P. camemberti have been deposited in the GenBank nucleotide core database under the accession codes HG793134 to HG793313. The EST sequence data of $P$. roquefort $i$ used for genome annotation have been deposited in the EMBL-EBI database under the accession codes HAAZ01000001-HAAZ01013080.

Supplementary Information accompanies this paper at http://www.nature.com/ naturecommunications

Competing financial interests: The authors declare no competing financial interests

Reprints and permission information is available online at http://www.npg.nature.com/ reprintsandpermissions/

How to cite this article: Cheeseman, K. et al. Multiple recent horizontal transfers of a large genomic region in cheese making fungi. Nat. Commun. 5:2876 doi: $10.1038 /$ ncomms3876 (2014).

This work is licensed under a Creative Commons AttributionNonCommercial-ShareAlike 3.0 Unported License. To view a copy of this license, visit http://creativecommons.org/licenses/by-nc-sa/3.0/ 